Check for updates

Cite this: Chem. Sci., 2018, 9, 681

\title{
Dynamics of recombination via conical intersection in a semiconductor nanocrystal $\dagger$
}

\begin{abstract}
Wei-Tao Peng, ${ }^{a}$ B. Scott Fales, (D) bc Yinan Shu (D) ${ }^{d}$ and Benjamin G. Levine (D) *a
Conical intersections are well known to introduce nonradiative decay pathways in molecules, but have only recently been implicated in nonradiative recombination processes in materials. Here we apply excited state ab initio molecular dynamics simulations based on a multireference description of the electronic structure to defective silicon nanocrystals up to $1.7 \mathrm{~nm}$ in diameter to search for accessible nonradiative recombination pathways. Dangling bond defects are found to induce conical intersections between the ground and first excited electronic states of five systems of various sizes. These defect-induced conical intersections are accessible at energies that are in the visible range (2.4-2.7 eV) and very weakly dependent on particle size. The dynamic simulations suggest that these intersections are accessed 40$60 \mathrm{fs}$ after creation of a defect-localized excitation. This ultrafast recombination is attributed to the fact that Jahn-Teller distortion on the first excited state drives the defect directly towards a conical intersection with the ground electronic state.
\end{abstract}

Received 27th September 2017 Accepted 13th November 2017

DOI: $10.1039 / \mathrm{c} 7 \mathrm{sc} 04221 \mathrm{c}$

rsc.li/chemical-science and other low-dimensional silicon systems have received intense attention due to their unique photophysical properties. Unlike bulk silicon, which has an indirect band gap, lowdimensional silicon materials can efficiently emit visible light ${ }^{19}$ with a wavelength that can be tuned via quantum confinement $^{2^{20-23}}$ or surface modification. ${ }^{24-27}$ This tunable emission enables their application in optoelectronic devices, ${ }^{28-30}$ biological imaging, ${ }^{31}$ and silicon lasers. ${ }^{32,33}$ Under ambient conditions, however, SiNCs are prone to oxidize quickly upon exposure to $\mathrm{O}_{2}$ and/or $\mathrm{H}_{2} \mathrm{O}$. Oxidation generates various defects on the surfaces of SiNCs, including dangling bonds (DBs) and some silicon oxide species ( $\mathrm{Si}-\mathrm{O}-\mathrm{Si}$ bridges and $\mathrm{Si}-\mathrm{OH}$ ). Among them, silicon dangling bond defects have been studied extensively. ${ }^{34-38}$ In general, there are two common types of dangling bond defects: $\mathrm{P}_{\mathrm{b}}$ centers, which are dangling bonds on a three-coordinated silicon atom on the surface, and D centers, which are DB defects located in amorphous silicon. ${ }^{39}$ DB defects have been known to degrade the performance of silicon-based devices for both photovoltaic ${ }^{\mathbf{4 0 , 4 1}}$ and light emission $^{\mathbf{3 8 , 4 2 - 4 4}}$ applications.

Thus, it is well-known that silicon DB defects are nonradiative centers in SiNCs. It is also established that electronic movement at DB centers is strongly coupled to local vibrational motions. ${ }^{45,46}$ The widely accepted mechanism for recombination involves the sequential capture of electron and hole into the non-bonding orbital of the $\mathrm{P}_{\mathrm{b}}$ center. Each change in the oxidation state of the $\mathrm{P}_{\mathrm{b}}$ center is accompanied by nuclear relaxation along a bending mode that maintains local $C_{3 \mathrm{v}}$ symmetry; reduction results in the defect silicon atom taking on a less planar structure (i.e. less $\mathrm{sp}^{2}$-like) while oxidation results in a more planar structure. The line of thinking that yields this
${ }^{a}$ Department of Chemistry, Michigan State University, East Lansing, MI 48824, USA. E-mail: levine@chemistry.msu.edu

${ }^{b}$ Department of Chemistry, The PULSE Institute, Stanford University, Stanford, CA 94305, USA

${ }^{c}$ SLAC National Accelerator Laboratory, Menlo Park, CA 94025, USA

${ }^{d}$ Department of Chemistry, University of Minnesota, Minneapolis, MN 55455, USA

$\dagger$ Electronic supplementary information (ESI) available. See DOI: $10.1039 / \mathrm{c} 7 \mathrm{sc} 04221 \mathrm{c}$ 
mechanism arises from the assumption that each change in charge state is instantaneous, however. It neglects (a) the fact that electrons and holes are strongly confined in SiNCs and therefore may interact strongly with one another and with the defect site before localization to the $\mathrm{P}_{\mathrm{b}}$ center, and (b) the interaction of electron and hole with the defect is not instantaneous and may, instead, involve complex electron-nuclear dynamics. The CI theory of recombination considers these interactions and dynamics explicitly, therefore it would be instructive to reinvestigate this recombination process from a CI point of view.

In this work we (a) investigate whether nonradiative dynamics at $\mathrm{P}_{\mathrm{b}}$ centers can be attributed to CIs between the ground and first excited electronic states, and (b) inform our fundamental physical intuition for recombination processes in general by analysis of the CIs associated with the dangling bond defect. To these ends we will bring to bear novel ab initio molecular dynamics (AIMD) tools capable of modeling the dynamics of electronically excited SiNCs as they approach CIs with the ground electronic state. In AIMD simulations, the nuclear dynamics are computed on PESs that are solved on the fly via electronic structure calculations. AIMD has recently become a tool of choice for the theoretical study of the photophysics of nanomaterials when either direct knowledge of excited state dynamics or extensive thermodynamic sampling are required, shedding light on various aspects of the charge carrier dynamics of SiNCs. ${ }^{47-56}$ The current study is the first to apply an AIMD approach based on a multireference description of the electronic structure to a true nanocrystal (diameter 1.7 $\mathrm{nm}$ ). The advantage of multireference electronic structure approaches such as the complete active space configuration interaction (CASCI) approach used here ${ }^{57,58}$ is that they can accurately describe the PES in the vicinity of CIs between the ground and first excited electronic states. This is in contrast to single reference electronic structure methods-such as timedependent density functional theory-which cannot accurately describe the potential energy surface near CIs involving the ground electronic state. ${ }^{59}$ The below study illustrates how applying CASCI-AIMD to model the dynamics of a semiconductor nanocluster from excitation to the neighborhood of a conical intersection can inform our fundamental understanding of nonradiative recombination.

\section{Results and discussion}

The methodological and computational specifics of our CASCIAIMD simulations are presented in ESI, $\dagger$ but here we outline our study. We have performed a single CASCI-AIMD simulation for each of a series of five silicon clusters. Each cluster has a single $P_{b}$ defect on the surface. The clusters (pictured in Fig. 1) range in size from a single sila-adamatane unit $\left(\mathrm{Si}_{10} \mathrm{H}_{15}\right)$ to a $1.7 \mathrm{~nm}$ particle $\left(\mathrm{Si}_{72} \mathrm{H}_{63}\right)$. All simulations were run on the first excited electronic PES starting from a structure in the FranckCondon region. (Structures are presented in ESI. $\dagger$ ) All surface silicon atoms aside from the defect site were capped with hydrogen atoms. We emphasize that these simulations are performed in the Born-Oppenheimer approximation.
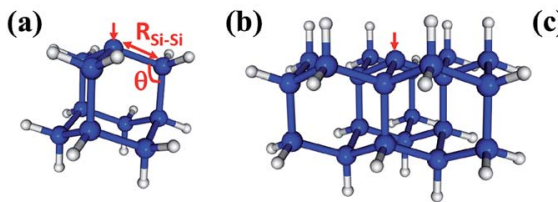

(c)

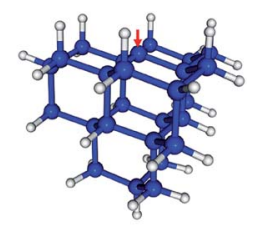

(d)
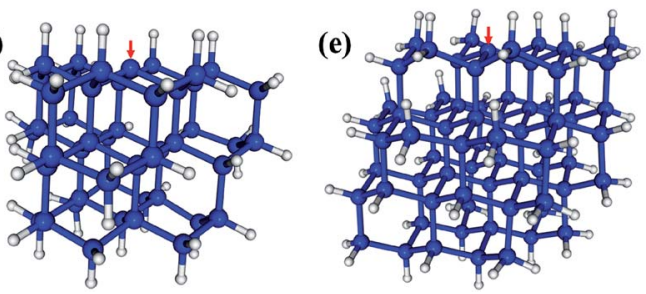

Fig. 1 The $\mathrm{P}_{\mathrm{b}}$-containing silicon clusters studied in this work. (a) $\mathrm{Si}_{10} \mathrm{H}_{15}$ (sila-adamantane cluster), (b) $\mathrm{Si}_{22} \mathrm{H}_{27}$, (c) $\mathrm{Si}_{26} \mathrm{H}_{31}$, (d) $\mathrm{Si}_{47} \mathrm{H}_{49}$ $(1.3 \mathrm{~nm} \mathrm{SiNC})$, and (e) $\mathrm{Si}_{72} \mathrm{H}_{63}(1.7 \mathrm{~nm} \mathrm{SiNC})$. One of the three symmetry-equivalent $\mathrm{Si}-\mathrm{Si}$ bond lengths $\left(R_{\mathrm{Si}-\mathrm{Si}}\right)$ and bond angles $(\theta)$ discussed herein are indicated in red in (a). The red arrows indicate the positions of dangling bond defects.

Geometries of near-zero energy gap were drawn from the AIMD trajectories and the minimal energy CIs (MECIs; the local minima on the CI seam) were optimized. Because CASCI lacks dynamic electron correlation, complete active space secondorder perturbation theory (CASPT2) calculations ${ }^{60}$ were performed on the smallest cluster to estimate errors in our CASCI energies. Static CASCI and CASCI-AIMD calculations were performed in the TeraChem software package, ${ }^{16,61-63}$ which enables these demanding calculations through the use of graphics processing units-high performance computer processors designed for graphical applications such as video games. CASPT2 calculations were performed in MolPro, ${ }^{64-68}$ coupled cluster calculations were performed in GAMESS, ${ }^{69-71}$ and conical intersection optimizations were performed with CIOpt. ${ }^{72}$ Through this work we prefer adiabatic state labels: $D_{0}$ and $D_{1}$ to indicate the ground and first excited spin doublet electronic states of the clusters, respectively. However, when useful and appropriate we also include term symbols ${ }^{2} \mathrm{~A}_{1}$ and ${ }^{2} \mathrm{E}$ to reflect the approximate symmetry of the states with respect to the local $C_{3 v}$ symmetry of the defect site.

The $\mathrm{D}_{0}\left({ }^{2} \mathrm{~A}_{1}\right)$ minimum energy structures of our five clusters are presented in Fig. 1. Bond lengths and bond angles around the $P_{b}$ defect (as indicated in Fig. 1a) at these structures are listed in the Table 1, labeled FC (Franck-Condon point). In these initial structures the three $\mathrm{Si}-\mathrm{Si}$ bond lengths surrounding the DB and the associated bond angles are symmetric. The vertical excitation energies (Table 1) of the five clusters are very similar to one another, varying by only $0.3 \mathrm{eV}$, and there is not a strong trend with system size. This absence of quantum confinement effects suggests the locality of the excitation. This locality can be seen in the orbitals involved in the excitation (pictured in Fig. 2). The excitation occurs from the highest doubly occupied molecular orbital (HDOMO) to the singly occupied molecular orbital (SOMO). The HDOMO is a SiSi $\sigma$ bonding orbital $\left(\sigma_{\mathrm{Si}-\mathrm{Si}}\right.$; e), which is relatively local to the region of the defect, while the SOMO is the dangling bond itself: 
Table 1 The Si-Si bond lengths ( $\mathrm{A})$, Si-Si-Si bond angles ( $\theta$ as indicated in Fig. 1a; in degrees), and $\mathrm{D}_{1}$ energies of differently sized silicon clusters at the Franck-Condon point (FC) and $\mathrm{MECl}$. All energies are relative to the $\mathrm{D}_{0}$ minimum energy. Energies are computed at the CASCI level of theory as described in ESI. When available, CASPT2 energies at CASCI geometries are presented in parenthesis

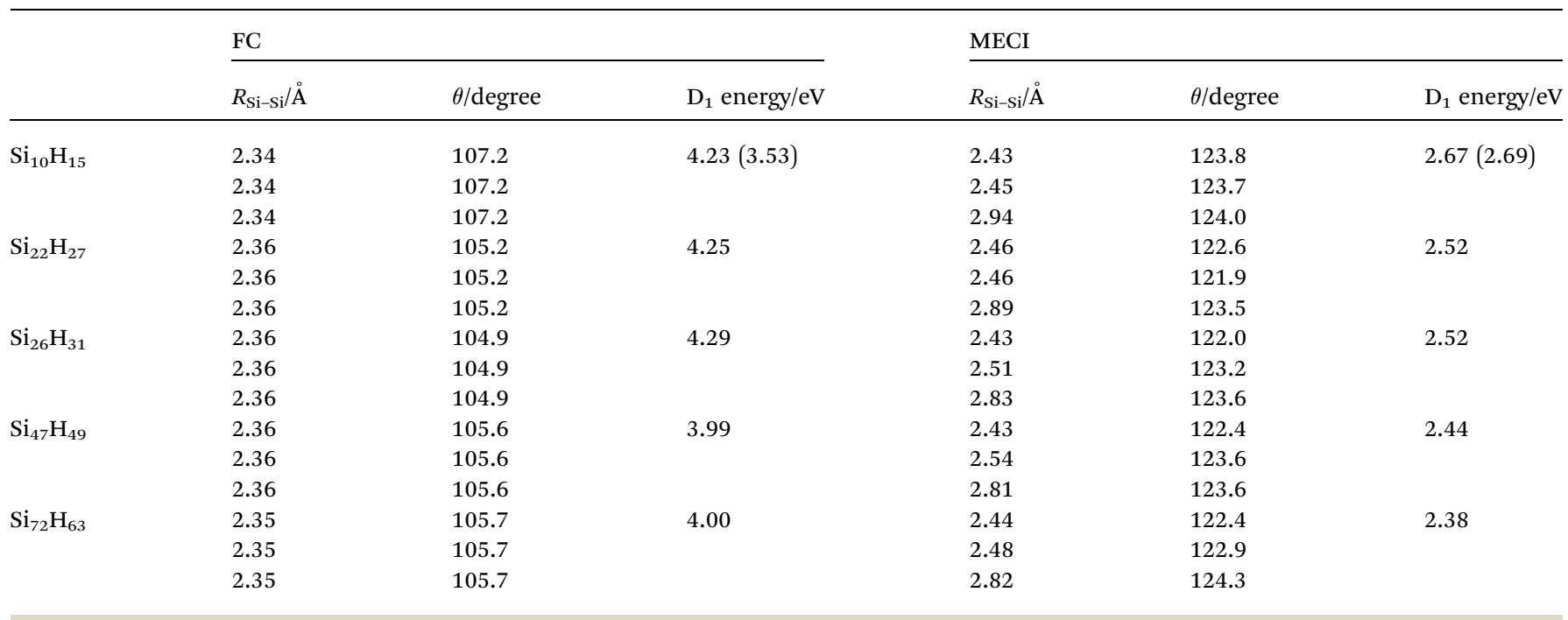

(a)

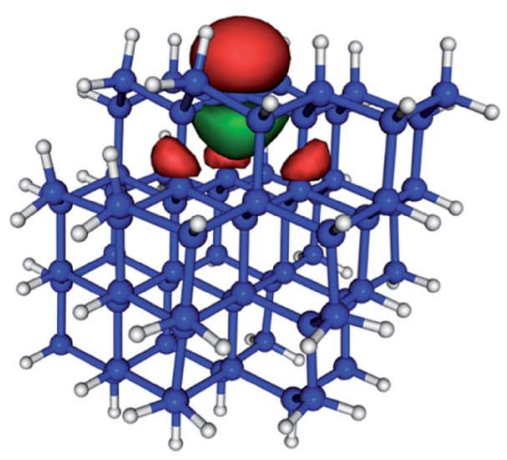

side view

(b)

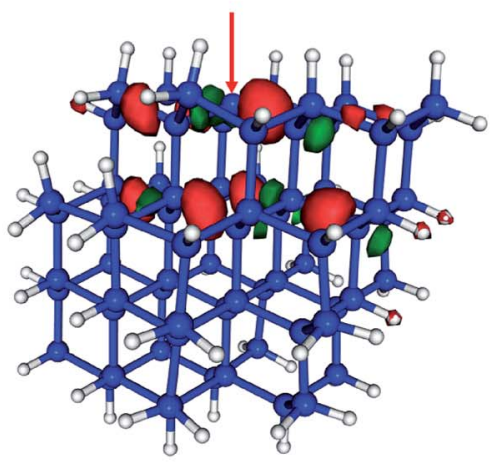

side view

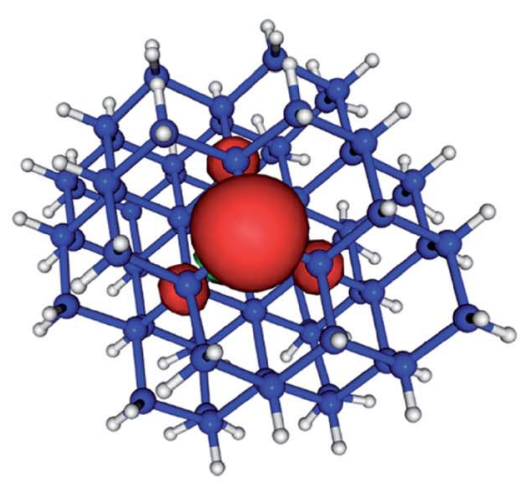

top view

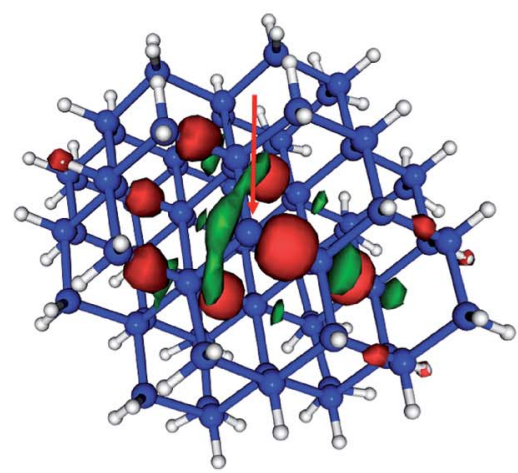

top view

Fig. 2 Orbitals representative of the $\mathrm{D}_{0} \rightarrow \mathrm{D}_{1}\left({ }^{2} \mathrm{~A}_{1} \rightarrow{ }^{2} \mathrm{E}\right.$ ) transition of the $\mathrm{Si}_{72} \mathrm{H}_{63}$ system. (a) The singly occupied molecular orbital (SOMO) is the nonbonding $(\mathrm{n})$ orbital of the $\mathrm{P}_{\mathrm{b}}$ center, and $(\mathrm{b})$ the highest doubly occupied orbital (HDOMO) has Si-Si $\sigma$ bonding character in the vicinity of the $\mathrm{P}_{\mathrm{b}}$ defect $\left(\sigma_{\mathrm{Si}-\mathrm{Si}_{\mathrm{i}}}\right)$. Note that this HDOMO is approximately degenerate due to the local symmetry of the defect. We show only one of the two nearly degenerate orbitals. The red arrows indicate the locations of the $\mathrm{P}_{\mathrm{b}}$ center. 
the nonbonding $\left(n ; a_{1}\right) \mathrm{sp}^{3}$ orbital of the defect silicon atom. Note that there is a nearly degenerate pair of HDOMOs due to the local $C_{3 \mathrm{v}}$ symmetry of the defect. We present only one of the two degenerate $\sigma_{\mathrm{Si}-\mathrm{Si}}$ orbitals in Fig. 2 .

Now we consider the results of the excited state AIMD simulations initiated on the $\mathrm{D}_{1}\left({ }^{2} \mathrm{E} ; \sigma_{\mathrm{Si}-\mathrm{Si}} \rightarrow \mathrm{n}\right)$ state. This is the lowest electronic state with nonzero transition dipole moment. Note that $D_{1}$ and $D_{2}$ are degenerate by symmetry, as will be discussed below, and $\mathrm{D}_{3}$ is considerably higher in energy $(0.9 \mathrm{eV}$ above $\mathrm{D}_{2}$ in the sila-adamantane cluster). The time-dependent $\mathrm{D}_{1}$ and $\mathrm{D}_{0}$ potential energies, $\mathrm{Si}-\mathrm{Si}$ bond lengths $\left(R_{\mathrm{Si}-\mathrm{Si}}\right.$ as defined in Fig. 1a), and bond angles ( $\theta$ as illustrated in Fig. 1a) of the $1.7 \mathrm{~nm}$ SiNC are presented in Fig. 3. The dynamics of the smaller clusters were nearly identical; similar graphs for these cases are reported in Fig. S1-S12. $\dagger$ In all five cases the $D_{1} / D_{0}$ energy gap approaches zero $(<0.1 \mathrm{eV})$ in the first $40-60$ fs after excitation (Fig. 3a). The vanishing energy gaps strongly suggest the existence of low-lying $\mathrm{D}_{1} / \mathrm{D}_{0}$ CIs. $^{73}$

Using low-gap structures from the AIMD trajectories as starting guesses, MECIs were optimized in all five systems. The energies and structural details of these MECIs are reported in Table 1. Full structures are presented in ESI. $\dagger$ Comparing the structures of MECIs to the Franck-Condon points, one can see that both bond lengths and bond angles around the DB defects increase at the MECIs. The three $\mathrm{Si}-\mathrm{Si}$ bonds surrounding the $\mathrm{DB}$ are asymmetrically stretched in all five clusters; one $\mathrm{Si}-\mathrm{Si}$ bond grows longer (2.81-2.94 angstrom) than the other two (2.43-2.54 angstrom). Similar asymmetric stretching is observed in the AIMD simulations of all five systems (Fig. 3b and S5-S8†) on the same 40-60 fs time scale on which the $D_{1} / D_{0}$ energy gap approaches zero. Much smaller changes in bond length are observed for Si-Si bonds not immediately adjacent to the $\mathrm{P}_{\mathrm{b}}$ defect. Consistent with past work on dangling bond defects, ${ }^{45}$ symmetric bending motion is also observed to be important; the $\theta$ angles are observed to increase significantly both in the AIMD simulations (Fig. 3c and S9-S12 $\dagger$ ) and in the optimized MECI structures (Table 1). Taken together, these calculations suggest that upon excitation of the lowest defect-localized excited state, the $P_{b}$ defect moves ballistically to the CI region in 40-60 fs. It is also noteworthy that the trajectories remain in a region of small energy gap after 40-60 fs, suggesting that it may pass over the intersection multiple times, enabling efficient decay.

The role that symmetry breaking plays in these nonradiative dynamics can be intuitively understood through analysis of the orbitals occupied during excitation. Fig. 4 is a schematic diagram summarizing these dynamics. As described above, excitation to $D_{1}$ involves the promotion of an electron from one of the degenerate $\sigma_{\mathrm{Si}-\mathrm{Si}}$ orbitals to the n orbital. This reduces the $\mathrm{Si}-\mathrm{Si}$ bond order, resulting in a lengthening of one of the $\mathrm{Si}-\mathrm{Si}$ bonds (as observed in the AIMD simulations of all five clusters). In the locally $C_{3 \mathrm{v}}$-symmetric FC structure the $\mathrm{D}_{1}\left({ }^{2} \mathrm{E}\right)$ state is doubly degenerate by symmetry, thus this symmetry-breaking motion is a Jahn-Teller distortion. The lengthening of a single Si-Si bond (moving from left to right along the $\mathrm{D}_{1}$ PES in Fig. 4) brings the molecule towards the MECI structure. This symmetry breaking raises the energy of one of the $\sigma_{\mathrm{Si}-\mathrm{Si}}$ orbitals into near degeneracy with the $\mathrm{n}$ orbital, bringing about a CI
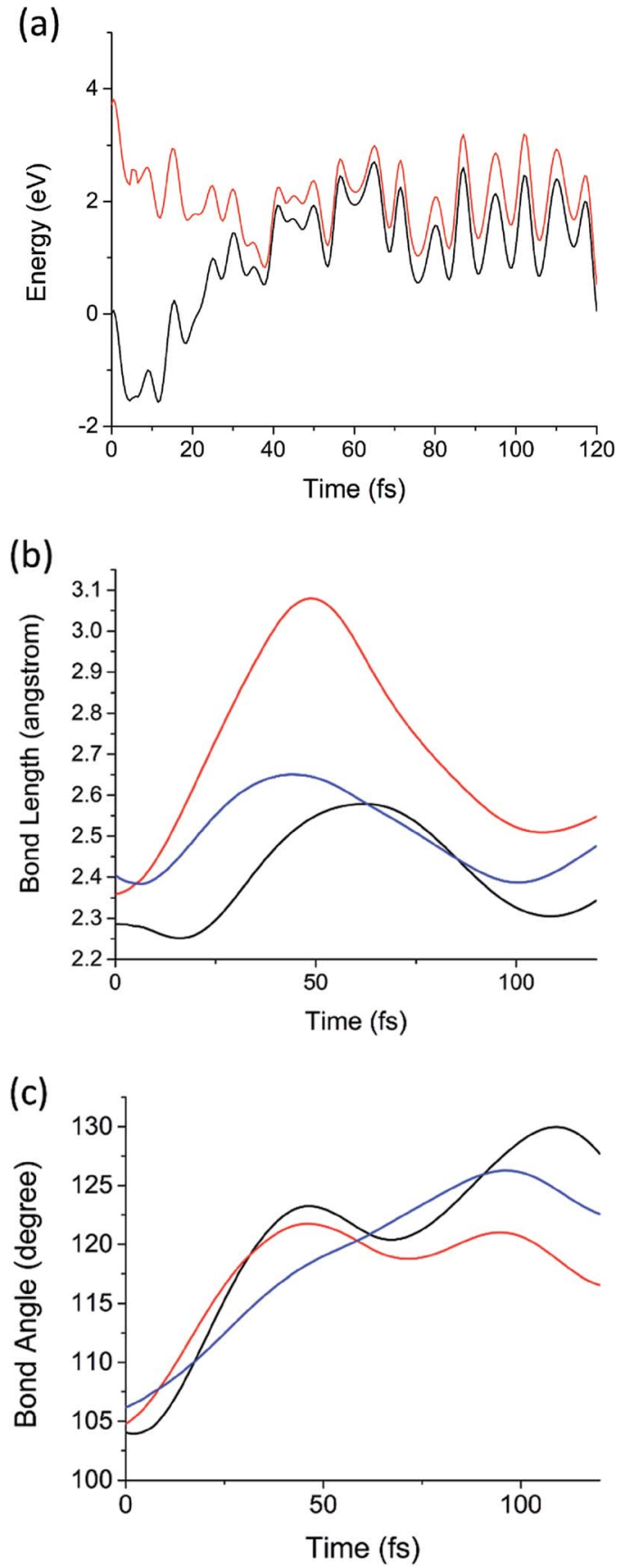

Fig. 3 (a) The potential energies of the $D_{1}$ (red) and $D_{0}$ (black) electronic states as a function of time from the $D_{1}$ AIMD simulation of $\mathrm{Si}_{72} \mathrm{H}_{63}$. (b) The three $\mathrm{Si}-\mathrm{Si}$ bond lengths $\left(R_{\mathrm{Si}-\mathrm{Si}}\right)$ adjacent to the $\mathrm{P}_{\mathrm{b}}$ defect as a function of time from the same AIMD calculation. (c) The three $\mathrm{Si}-\mathrm{Si}-\mathrm{Si}$ angles $(\theta$, illustrated in Fig. 1a) as a function of time from the same AIMD calculation. Each color represents one of the three symmetry equivalent bond lengths or angles in (b) and (c), respectively.

between the $D_{0}$ and $D_{1}$ states. That a Jahn-Teller distortion in $\mathrm{D}_{1}$ drives the molecules directly towards the $\mathrm{D}_{1} / \mathrm{D}_{0}$ CI provides a straightforward explanation for the ultrafast nonradiative process that follows creation of the defect-localized excitation.

The MECIs of the defective silicon clusters studied here are all accessible at energies in the visible range (2.38-2.67 eV above the ground state minimum structure; Table 1) and therefore 


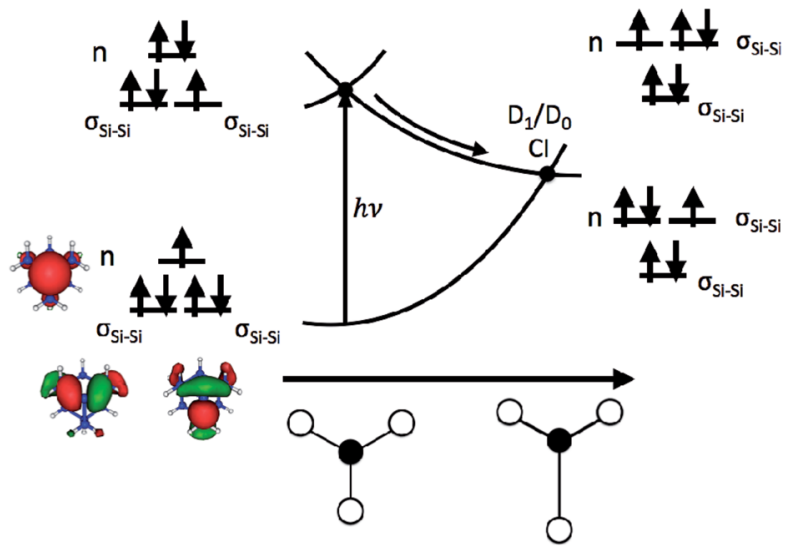

Fig. 4 Schematic illustration of the dynamics of nonradiative recombination of an excitation at a $\mathrm{P}_{\mathrm{b}}$ center. The PESs are plotted as a function of an asymmetric stretching coordinate about the $P_{b}$ center (illustrated along the $x$-axis with the dangling bond site represented by a filled circle and the three adjacent silicon atoms represented by open circles). Insets show the orbital occupations of $D_{0}$ and $D_{1}$ and relative orbital energies at the FC point (left) and $\mathrm{MECl}$ (right). The $\mathrm{n}$ and $\sigma_{\mathrm{Si}-\mathrm{Si}}$ orbitals of the smallest (sila-adamantane) system are shown on the bottom left.

capable of quenching visible light emission. The MECIs show a slight decrease in energy with increasing system size; the energy decreases from $2.67 \mathrm{eV}$ for the small sila-adamantane cluster to $2.38 \mathrm{eV}$ for the $1.7 \mathrm{~nm}$ SiNC. This small energy decrease of $0.29 \mathrm{eV}$ is consistent with the localized nature of the defect-localized excited state. Calculations at the dynamically correlated CASPT2 level confirm the accuracy of the MECI energies predicted by CASCI, though vertical excitation energies are somewhat overestimated. All five clusters have $\mathrm{D}_{1}$ minimum energy structures distinct from the MECIs. In all cases this minimum is $0.05-0.06 \mathrm{eV}$ below the MECI, compared to the 1.6$1.9 \mathrm{eV}$ released during relaxation on the excited states. Thus the MECI is energetically accessible upon excitation. Energies and structures of $\mathrm{D}_{1}$ minima are presented in ESI. $\dagger$

The existence of defect-induced CIs with energies in the 2.4$2.7 \mathrm{eV}$ range is consistent with several experimental observations of the photoluminescence (PL) of SiNCs after oxidation. The MECI energies suggest that the PL of SiNCs with emission energies larger than $\sim 2.4-2.7 \mathrm{eV}$ is likely to be quenched by the DB defects. Indeed the quantum yield of PL from oxidized SiNCs is observed to drop with increasing energy, ${ }^{74}$ and single particle experiments on oxidized SiNCs show no emissive particles with PL maxima above $2.5 \mathrm{eV} \cdot{ }^{75}$ In addition, the PL lifetime of oxidized SiNCs decreases with increasing PL energy, and a dramatic decrease is observed in the $2.0-2.2 \mathrm{eV}$ energy range. ${ }^{76}$ This decreasing PL lifetime suggests the existence of an efficient nonradiative recombination pathway accessible above these energies, consistent with our computed CI energies. Finally, as noted in our previous studies of oxygen-containing defects, the unusual size-insensitive orange (S-band) emission of oxidized SiNCs observed in ensemble PL measurements ${ }^{77}$ is consistent with the presence of CIs accessible in this energy range. We argue that the size-insensitivity of the observed ensemble emission arises not because the emission energy of individual oxidized SiNCs becomes insensitive to particle size, but instead because the rate of nonradiative recombination is strongly size sensitive, dramatically reducing the PL yields of smaller SiNCs with shorter wavelengths. This argument reconciles the observation of size-insensitive emission with PL lifetime, linewidth, and polarization measurements suggesting that the S-band arises from quantum-confined excitons. ${ }^{75,78,79}$

\section{Conclusions}

Thus, we have elucidated the mechanism of nonradiative recombination via a dangling bond defect by application of AIMD simulations based on a multireference description of the electronic structure to SiNCs up to $1.7 \mathrm{~nm}$ in diameter. Within 40-60 fs after excitation of a defect-localized electronic excited state, a CI between the $\mathrm{D}_{0}$ and $\mathrm{D}_{1}$ states is accessed. This CI is accessible at energies in the $2.4-2.7 \mathrm{eV}$ range, and thus is detrimental to visible PL, consistent with the fact that DBs are well-known nonradiative centers. The ultrafast recombination process is driven both by Jahn-Teller distortion in the $\mathrm{D}_{1}$ state and by totally symmetric bending of the $\mathrm{DB}$ center. The role that symmetry-breaking plays in this mechanism underlines the importance of treating coupled electron-nuclear dynamics in the study of recombination.

\section{Conflicts of interest}

There are no conflicts to declare.

\section{Acknowledgements}

We gratefully acknowledge support from the National Science Foundation under grant CHE-1565634. We acknowledge fruitful discussion with Rémi Beaulac.

\section{References}

$1 \mathrm{~J}$. Michl and V. Bonacic-Koutecky, Electronic Aspects of Organic Photochemistry, Wiley, New York, 1990.

2 D. R. Yarkony, Rev. Mod. Phys., 1996, 68, 985-1013.

3 F. Bernardi, M. Olivucci and M. A. Robb, Chem. Soc. Rev., 1996, 25, 321-328.

4 B. G. Levine and T. J. Martinez, Annu. Rev. Phys. Chem., 2007, 58, 613-634.

5 S. Matsika and P. Krause, Annu. Rev. Phys. Chem., 2011, 62, 621-643.

6 D. R. Yarkony, Chem. Rev., 2012, 112, 481-498.

7 R. Gonzalez-Luque, M. Garavelli, F. Bernardi, M. Merchan, M. A. Robb and M. Olivucci, Proc. Natl. Acad. Sci. U. S. A., 2000, 97, 9379-9384.

8 M. L. Hause, Y. H. Yoon, A. S. Case and F. F. Crim, J. Chem. Phys., 2008, 128, 104307-104309.

9 K. R. Yang, X. F. Xu, J. J. Zheng and D. G. Truhlar, Chem. Sci., 2014, 5, 4661-4680.

10 X. L. Zhu and D. R. Yarkony, J. Chem. Phys., 2014, 140, 21.

11 D. Polli, P. Altoe, O. Weingart, K. M. Spillane, C. Manzoni, D. Brida, G. Tomasello, G. Orlandi, P. Kukura, 
R. A. Mathies, M. Garavelli and G. Cerullo, Nature, 2010, 467, 440-U488.

12 M. Liebel, C. Schnedermann and P. Kukura, Phys. Rev. Lett., 2014, 112, 198302.

13 S. Matsika, J. Phys. Chem. A, 2004, 108, 7584-7590.

14 M. Merchan and L. Serrano-Andres, J. Am. Chem. Soc., 2003, 125, 8108-8109.

15 I. Schapiro, F. Melaccio, E. N. Laricheva and M. Olivucci, Photochem. Photobiol. Sci., 2011, 10, 867-886.

16 B. S. Fales and B. G. Levine, J. Chem. Theory Comput., 2015, 11, 4708-4716.

17 Y. Shu, B. S. Fales and B. G. Levine, Nano Lett., 2015, 15, 6247-6253.

18 Y. Shu, B. S. Fales, W.-T. Peng and B. G. Levine, J. Phys. Chem. Lett., 2017, 8, 4091-4099.

19 L. T. Canham, Appl. Phys. Lett., 1990, 57, 1046-1048.

20 V. Kocevski, O. Eriksson and J. Rusz, Phys. Rev. B: Condens. Matter Mater. Phys., 2013, 87, 245401.

21 M. L. Mastronardi, F. Hennrich, E. J. Henderson, F. MaierFlaig, C. Blum, J. Reichenbach, U. Lemmer, C. Kubel, D. Wang, M. M. Kappes and G. A. Ozin, J. Am. Chem. Soc., 2011, 133, 11928-11931.

22 B. Delley and E. F. Steigmeier, Phys. Rev. B: Condens. Matter Mater. Phys., 1993, 47, 1397-1400.

23 S. Furukawa and T. Miyasato, Phys. Rev. B: Condens. Matter Mater. Phys., 1988, 38, 5726-5729.

24 Z. Y. Zhou, L. Brus and R. Friesner, Nano Lett., 2003, 3, 163167.

25 D. Konig, J. Rudd, M. A. Green and G. Conibeer, Sol. Energy Mater. Sol. Cells, 2009, 93, 753-758.

26 M. Dasog, G. B. De los Reyes, L. V. Titova, F. A. Hegmann and J. G. C. Veinot, ACS Nano, 2014, 8, 9636-9648.

27 H. S. Li, Z. G. Wu, T. L. Zhou, A. Sellinger and M. T. Lusk, Phys. Chem. Chem. Phys., 2014, 16, 19275-19281.

28 B. Gelloz and N. Koshida, J. Appl. Phys., 2000, 88, 4319-4324.

29 C. Y. Liu, Z. C. Holman and U. R. Kortshagen, Nano Lett., 2009, 9, 449-452.

30 J. Valenta, N. Lalic and J. Linnros, Opt. Mater., 2001, 17, 4550.

31 J. H. Park, L. Gu, G. von Maltzahn, E. Ruoslahti, S. N. Bhatia and M. J. Sailor, Nat. Mater., 2009, 8, 331-336.

32 L. Pavesi, L. Dal Negro, C. Mazzoleni, G. Franzo and F. Priolo, Nature, 2000, 408, 440-444.

33 D. Liang and J. E. Bowers, Nat. Photonics, 2010, 4, 511-517.

34 M. A. Tischler, R. T. Collins, J. H. Stathis and J. C. Tsang, Appl. Phys. Lett., 1992, 60, 639-641.

35 C. Delerue, G. Allan and M. Lannoo, Phys. Rev. B: Condens. Matter Mater. Phys., 1993, 48, 11024-11036.

36 M. Schoisswohl, J. L. Cantin, H. J. Vonbardeleben and G. Amato, Appl. Phys. Lett., 1995, 66, 3660-3662.

37 R. N. Pereira, D. J. Rowe, R. J. Anthony and U. Kortshagen, Phys. Rev. B: Condens. Matter Mater. Phys., 2011, 83, 155327.

38 N. P. Brawand, M. Voros and G. Galli, Nanoscale, 2015, 7, 3737-3744.

39 E. H. Poindexter, P. J. Caplan, B. E. Deal and R. R. Razouk, J. Appl. Phys., 1981, 52, 879-884.
40 S. Niesar, A. R. Stegner, R. N. Pereira, M. Hoeb, H. Wiggers, M. S. Brandt and M. Stutzmann, Appl. Phys. Lett., 2010, 96, 193112.

41 H. S. Li, Z. G. Wu and M. T. Lusk, J. Phys. Chem. C, 2014, 118, 46-53.

42 B. K. Meyer, V. Petrovakoch, T. Muschik, H. Linke, P. Omling and V. Lehmann, Appl. Phys. Lett., 1993, 63, 1930-1932.

43 B. G. Fernandez, M. Lopez, C. Garcia, A. Perez-Rodriguez, J. R. Morante, C. Bonafos, M. Carrada and A. Claverie, J. Appl. Phys., 2002, 91, 798-807.

44 X. D. Pi, L. Mangolini, S. A. Campbell and U. Kortshagen, Phys. Rev. B: Condens. Matter Mater. Phys., 2007, 75, 177.

45 C. Delerue and M. Lannoo, Nanostructure: Theory and Modelling, Springer-Verlag, Berlin, 2004.

46 M. Lannoo, C. Delerue and G. Allan, J. Lumin., 1993, 57, 243247.

47 J. C. Chen, A. Schmitz, T. Inerbaev, Q. G. Meng, S. Kilina, S. Tretiak and D. S. Kilin, J. Phys. Chem. Lett., 2013, 4, 2906-2913.

48 J. Liu, A. J. Neukirch and O. V. Prezhdo, J. Chem. Phys., 2013, 139, 164303.

49 Y. Shu and B. G. Levine, J. Chem. Phys., 2013, 139, 081102.

50 J. Liu, A. J. Neukirch and O. V. Prezhdo, J. Phys. Chem. C, 2014, 118, 20702-20709.

51 Y. Shu and B. G. Levine, J. Phys. Chem. C, 2014, 118, 76697677.

52 Y. Han, D. A. Micha and D. S. Kilin, Mol. Phys., 2015, 113, 327-335.

53 K. G. Reeves, A. Schleife, A. A. Correa and Y. Kanai, Nano Lett., 2015, 15, 6429-6433.

54 Y. Shu and B. G. Levine, J. Phys. Chem. C, 2015, 119, 17371747.

55 Y. Shu and B. G. Levine, J. Phys. Chem. C, 2016, 120, 2324623253.

56 N. P. Brawand, M. B. Goldey, M. Voros and G. Galli, Chem. Mater., 2017, 29, 1255-1262.

57 G. Granucci, M. Persico and A. Toniolo, J. Chem. Phys., 2001, 114, 10608-10615.

58 P. Slavicek and T. J. Martinez, J. Chem. Phys., 2010, 132, 234102.

59 B. G. Levine, C. Ko, J. Quenneville and T. J. Martinez, Mol. Phys., 2006, 104, 1039-1051.

60 J. Finley, P. A. Malmqvist, B. O. Roos and L. Serrano-Andres, Chem. Phys. Lett., 1998, 288, 299-306.

61 I. S. Ufimtsev and T. J. Martinez, J. Chem. Theory Comput., 2009, 5, 2619-2628.

62 E. G. Hohenstein, M. E. F. Bouduban, C. C. Song, N. Luehr, I. S. Ufimtsev and T. J. Martinez, J. Chem. Phys., 2015, 143, 014111.

63 C. C. Song, L. P. Wang and T. J. Martinez, J. Chem. Theory Comput., 2016, 12, 92-106.

64 P. J. Knowles and H. J. Werner, Chem. Phys. Lett., 1985, 115, 259-267.

65 H.-J. Werner and P. J. Knowles, J. Chem. Phys., 1985, 82, 5053-5063.

66 H. J. Werner, Mol. Phys., 1996, 89, 645-661. 
67 P. Celani and H. J. Werner, J. Chem. Phys., 2000, 112, 55465557.

68 H. J. Werner, P. J. Knowles, G. Knizia, F. R. Manby and M. Schutz, Wires Comput. Mol. Sci., 2012, 2, 242-253.

69 M. W. Schmidt, K. K. Baldridge, J. A. Boatz, S. T. Elbert, M. S. Gordon, J. H. Jensen, S. Koseki, N. Matsunaga, K. A. Nguyen, S. Su, T. L. Windus, M. Dupuis and J. A. Montgomery, J. Comput. Chem., 1993, 14, 1347-1363.

70 J. R. Gour, P. Piecuch and M. Wloch, J. Chem. Phys., 2005, 123, 134113.

71 J. R. Gour and P. Piecuch, J. Chem. Phys., 2006, 125, 234107.

72 B. G. Levine, J. D. Coe and T. J. Martinez, J. Phys. Chem. B, 2008, 112, 405-413.

73 D. G. Truhlar and C. A. Mead, Phys. Rev. A: At., Mol., Opt. Phys., 2003, 68, 032501.
74 D. Jurbergs, E. Rogojina, L. Mangolini and U. Kortshagen, Appl. Phys. Lett., 2006, 88, 233116.

75 T. Schmidt, A. I. Chizhik, A. M. Chizhik, K. Potrick, A. J. Meixner and F. Huisken, Phys. Rev. B: Condens. Matter Mater. Phys., 2012, 86, 125302.

76 F. Huisken, G. Ledoux, O. Guillois and C. Reynaud, Adv. Mater., 2002, 14, 1861-1865.

77 M. V. Wolkin, J. Jorne, P. M. Fauchet, G. Allan and C. Delerue, Phys. Rev. Lett., 1999, 82, 197-200.

78 C. Garcia, B. Garrido, P. Pellegrino, R. Ferre, J. A. Moreno, J. R. Morante, L. Pavesi and M. Cazzanelli, Appl. Phys. Lett., 2003, 82, 1595-1597.

79 I. Sychugov, R. Juhasz, J. Valenta and J. Linnros, Phys. Rev. Lett., 2005, 94, 087405. 